\title{
Dynamic integrity constraints definition and enforcement in databases: a classification framework
}

\author{
M. A. Pacheco e Silva* \\ Universitat Politècnica de Catalunya \\ Departament de Llenguatges i Sistemes Informàtics \\ Campus Nord - Jordi Girona Salgado 1-3 \\ 08034 - Barcelona - Spain \\ Tel.: + $3434017332 \quad$ Fax: +3434017014 \\ E-mail:pacheco@lsi.upc.es
}

\begin{abstract}
A dynamic constraint expresses conditions that involve facts of two or more states of the database. Since the database is expected to satisfy such conditions, a fundamental issue about integrity constraints is their enforcement. Many methods have been developed in this respect. The purpose of this paper is to present a brief survey of methods for dynamic constraint definition and enforcement. Also we classify and compare these methods with respect to several relevant features.
\end{abstract}

\section{Keywords}

Dynamic integrity constraints, integrity enforcement, survey, classification

\footnotetext{
"Permanent address: Diretoria de Processamento de Dados, Universidade Federal de Uberlândia, Uberlândia, MG, Brazil (E-mail: mamelia@pitanga.ufu.br)
} 


\section{INTRODUCTION}

In the field of information systems and databases, the term integrity normally refers to the correctness or validity of the stored data, as defined explicitly by means of integrity rules or integrity constraints (Grefen and Apers, 1993). Integrity is a very important property of information systems. The lack of integrity has usually negative consequences (Olivé, 1995).

Motro (1989) makes the distinction between two components of integrity: validity and completeness. We can say that a database has integrity if all its data are correct (valid) and if it contains all relevant data (it is complete). In particular, completeness is a necessary condition when one takes the closed world assumption: in this case, lack of completeness may lead the system to deduce facts that are not valid.

In general, neither the full correctness with respect to the part of the real world modeled by the database is guaranteed, nor the completeness of the facts stored in the database is ensured (Motro, 1989; Grefen and Apers, 1993). For most systems, full integrity can only be achieved with human supervision, because the system cannot determine if a fact is true or false and it cannot detect changes in the Universe of Discourse (UD). To ensure integrity, one should check systematically the facts with respect to the UD.

However it is possible to build some mechanisms to guarantee some level of integrity in a database. This is achieved by means of conditions defined over the facts of the database called integrity constraints (or, for short, constraints). If these conditions are satisfied, we may have some level of confidence on the database integrity (Olivé, 1995). Therefore the integrity of a database is partially guaranteed by means of integrity constraints, i.e. conditions that define properties to be satisfied by the database.

Constraints are conceptually divided into two classes: inherent and explicit constraints. The former express restrictions due to the semantics of the data model, i.e. they are implicit in the database schema. The latter are arbitrary properties to be satisfied by the database that cannot be captured by schema restrictions (Fraternali and Paraboschi, 1993). Then they must be explicitly expressed as a formula, generally a first-order logic formula.

Constraints also can be distinguished as static (or state) and dynamic integrity constraints. A static constraint expresses state-independent properties that must hold at any state of the database. It depends only on the current state, independently of any previous states of the database (Plexousakis, 1991). An example of static constraint is the condition "an employee's salary must be less than her manager's". A dynamic constraint allows expressing conditions over (usually time-ordered) sequence of two or more database states, e.g. the condition "an employee's salary must never decrease". There is a particular case of dynamic 
constraints called transition constraints. A transition constraint imposes restrictions on pairs of states, the before and after state of a transaction (Fraternali and Paraboschi, 1993).

Since the database is expected to be consistent with respect to these properties, one important issue is the enforcement of integrity constraints upon updates. Integrity enforcement deals with the prevention of semantic errors made by users due to their carelessness or lack of knowledge (Grefen and Apers, 1993).

Despite the extensive research conducted during the last decade, constraint enforcement has yet to become a practical technology. This is due to the lack of efficient methods for checking the satisfaction of general constraints. Commercial database management systems only provide automatic enforcement of limited types of constraints, such as keys and referential integrity constraints (Plexousakis, 1995).

An issue related to integrity constraint management includes constraint verification. This is a process to check if the constraints themselves are valid syntactically and semantically. Constraint verification includes several issues as shown by Grefen and Apers (1993). One of these issues is constraint satisfiability that consists of determining whether the set of constraints is a satisfiable set. This problem is important since it would be useful to know if the defined set of constraints is satisfiable before trying to enforce their satisfaction. However this topic is out of the scope of this paper. We suppose that the constraints are satisfiable and correct. Works about constraint satisfiability can be found in Bry et al. (1988) and Formica et al. (1994).

Our objective is to review the research on constraint enforcement in database systems. We are mainly interested on methods developed in the context of dynamic integrity constraints. The paper is organized as follows. Section 2 introduces some general concepts of database constraint enforcement and describes some works in the scope of static integrity constraints. Section 3 describes several contributions to dynamic constraint enforcement and compares them with respect to a number of characterizing features. Section 4 presents the conclusion of the paper.

\section{STATIC INTEGRITY CONSTRAINT ENFORCEMENT}

Static constraints have been studied since the beginning of databases. The first methods for static constraint enforcement were developed for relational databases, dealing with restricted classes of constraints. In this section we discuss some methods of static constraint enforcement. Some state-of-the-art surveys in this topic can be consulted in Bry et al. (1990), Plexousakis (1991), Fraternali and Paraboschi (1993) and Grefen and Apers (1993).

One of the earliest works addressing this issue is the paper of Florentin (1974). This paper comments that the consistency of the stored data in databases can be 
preserved through the statement of logical constraints. The author remarks that integrity can best be maintained by checking the constraints when an update is carried out. Moreover he indicates the possibility of using a kind of theorem prover to derive simplified constraints, but rejects the feasibility of such an approach in practice.

A different approach, also in the scope of relational databases, is taken by Stonebraker (1975), where the formalism of the query language QUEL is used to formulate a way of treating a restricted class of integrity constraint with more automatization. Nicolas (1982) was pioneer in extending the class of constraints into any closed first-order formula. Since then, many methods for integrity enforcement of these constraints have been developed.

There has been a large cumulative effort to achieve the static constraint enforcement. Many methods have been developed with this objective. These methods differ in the kind of databases considered (for example, relational, deductive or object-oriented), in the kind of updates they consider (only single insertions, deletions or modifications of facts, or compound updates) and in the kind of enforcement they carry on (integrity checking or integrity maintenance).

Integrity checking is the process of verifying that a given update satisfies the constraints. If any constraint is violated, then the update is rejected. Otherwise the update is accepted. Integrity maintenance is a process that also starts with a given update and the constraints but now, if some constraint is violated, an attempt is made to find a repair, that is, an additional set of insertions and/or deletions of facts to be added to the update, such that the resulting update satisfies all integrity constraints (Teniente and Olivé, 1995).

Based on the time in which the method can be applied to enforce the constraints, there are two approaches. The first is a run-time approach, that must be carried out in the time the update takes place. It takes into account the information provided by the update request and the contents of the database. The second is a compiletime approach, that is based on meeting a solution to enforce constraints at compile-time, i.e. before the time of update execution. In this case, production rules, ECA (Event-Condition-Action) rules or transaction programs are generated from a database schema and a proposed update description.

The simplest solution of static constraint enforcement would be to evaluate all constraints whenever the database is updated. However this naive approach is impractical, because it is too costly and highly redundant. To avoid such situation all practical methods are based on the premise that constraints are known to be satisfied prior to the update. Only a subset of the constraints need to be verified after the update, namely those that are affected by it. Then, given a particular update, methods derive simplified conditions of the constraints such that, if the database satisfies the simplified conditions, it is guaranteed that the database will be consistent after the update (Olivé, 1991).

Many recent works have proposed that the technology of active databases provides a natural framework for implementing integrity enforcement through 
repairing actions (Ceri and Widom, 1990; Fraternali and Paraboschi, 1993; Ceri et al., 1994b). The predominant paradigm for active databases is that of ECA rules which are triggered by an event and their action is executed only if a condition is met. Nowadays, works in the field of active databases investigate the rule-based integrity maintenance.

Next we consider three criteria to group some methods for static constraint enforcement:

- Kind of the database considered: some examples in the area of relational databases are Nicolas (1982), Henschen et al. (1984), Hsu and Imielinski (1985), Ceri and Widom (1990), Widom et al. (1994). The methods developed by Decker (1986), Kowalski et al. (1987), Lloyd et al. (1987), Moerkotte and Lockemann (1991), Wüthrich (1993), Pastor and Olivé (1994), Asirelli et al. (1996), Lee and Ling (1996) deal with deductive databases. In the area of object-oriented databases we can refer the works of Jeusfeld and Jarke (1991), Palopoli and Torlone (1992), Siebes and Van der Voort (1992), Karadimce and Urban (1993). Examples in the area of active databases are the methods developed by Siebes and Van der Voort (1992), Karadimce and Urban (1993), Ceri et al. (1994b). Deductive object-oriented databases are considered by Urban and Delcambre (1990), Caseau (1991), Ceri et al. (1994a), Bertino et al. (1997).

- Kind of the constraint enforcement: if a method is an integrity checking method or an integrity maintenance method. Some integrity checking methods are described by Hsu and Imielinski (1985), Kowalski et al. (1987), Lloyd et al. (1987), Bry et al. (1988), Urban and Delcambre (1990), Bertino et al. (1997). The works presented by Ceri and Widom (1990), Moerkotte and Lockemann (1991), Palopoli and Torlone (1992), Karadimce and Urban (1993), Wüthrich (1993), Ceri et al. (1994b) are integrity maintenance methods.

- Time in which the method can be applied to enforce the constraints: if it is a run-time or a compile-time method. Some methods with run-time approach are Kowalski et al. (1987), Moerkotte and Lockemann (1991), Palopoli and Torlone (1992), Wüthrich (1993), Teniente and Olivé (1995). Examples of compile-time methods are Ceri and Widom (1990), Wallace (1991), Ceri et al. (1994b), Pastor and Olivé (1994), Widom et al. (1994).

Other criteria to group constraint enforcement methods have been presented in the literature. For example, one of the criteria that Grefen and Apers (1993) take into account is the constraint enforcement strategy employed by the method: violation prevention before the database is actually updated or violation detection after the database has been updated. Another criterion we could consider is whether the method requires total or partial information. Normally, methods of checking a constraint for a modification require total information, i.e. they need to access the constraint specification, the modification and all the existing data relevant to the 
constraint. An example of a partial information method is the work of Widom et al. (1994). They explore a method that requires only some of this information: the constraint specifications only; the constraint specifications and the modified data; or the constraint specification, the modified data and portions of the existing data.

\section{DYNAMIC INTEGRITY CONSTRAINT ENFORCEMENT}

As commented before, a dynamic integrity constraint involves facts of two or more states of the database. Sometimes it involves only facts of two consecutive states, constraining the transition between them (a transition constraint, in this case), but a general dynamic constraint may refer to any number of states.

Most researches in integrity enforcement deal with static constraints, whereas only relatively few papers have dealt with handling of transition or dynamic constraints because the problem is more complex. It requires expensive reasoning in multiple database states and has an inherent connection to the area of temporal logic and temporal reasoning (Plexousakis, 1991).

An early work dealing with dynamic constraint checking is Nicolas and Yazdanian (1978). They present a method in the scope of deductive databases. Static and dynamic (transition, in fact) constraints in the form of clauses are discussed and the issues involved in working with constraints together with derivation rules in one system are addressed.

Qian and Waldinger (1988) recognize the need for efficient checking of dynamic constraints by means of a method with less amount of history maintained. Their formalism is based on a variant of first-order situational logic in which the states of computations are explicit objects. Integrity constraints and transactions are uniformly specifiable as expressions in their language. The paper presents many compelling examples but no general method.

Due to the intimate connection of the problem of dynamic constraint enforcement with temporal reasoning, most of the methods developed employ some form of temporal logic (Lipeck and Saake, 1987; Chomicki, 1992; Bidoit and De Amo, 1994; Sistla and Wolfson, 1995). Almost all languages that allow defining dynamic constraints use temporal logic. Only a few languages use explicit time to express dynamic constraints. Among them are CIAM (Gustafsson et al., 1982), Telos (Mylopoulos et al., 1990), ODISSEA (Sancho and Olivé, 1993) and ROSES (Pacheco and Sancho, 1997).

In this section we review several methods of dynamic constraint enforcement. These methods differ in many aspects. In order to establish a point of reference among the approaches, we introduce some features that allow us to classify and to make a comparison of them:

- Kind of dynamic constraint: this feature indicates if the method deals with general dynamic constraints or if it only considers specific kinds as, for 
example, transition constraints or dynamic dependencies. General dynamic constraints and transition constraints have been defined before. Dynamic dependencies will be commented for the approach of Bidoit and De Amo (1994).

- Kind of the database considered: the kind of the database used as basis to develop the method. In this paper, we present methods related with the following kinds of databases: relational, deductive, object-oriented (OO), deductive object-oriented (DOO), active and temporal databases.

- Kind of constraint enforcement: which indicates if the method proposes checking or maintenance of the database integrity. We consider that the method is a maintenance approach if the integrity violation can be safely repaired, either by modifying the original transaction or by post-processing it. If the response given by the method to constraint violation is only to reject or rollback the transaction, we consider it a checking method. Generally, methods of enforcing dynamic constraints only perform checking of the constraint violation. It means that most methods are not prepared for repairing the database consistency if some constraint is violated.

- Time of constraint enforcement: this feature refers to the instant in which the method is applied. Enforcement can be performed completely at compile-time, completely at run-time, or partially at compile-time and partially at run-time. The majority of the methods in the literature are run-time methods. However, (completely or partially) compile-time methods have the advantage over runtime methods because performance is less critical at compile-time.

- Constraint language used: the language utilized in the method to specify dynamic constraints. In the case of transition constraints, it is possible to use a first-order logic formula, the same commonly used for static constraints, extended by operators such as old or new. However, for the specification of general dynamic constraints, it is necessary to use a first-order logic extended with temporal quantifiers (temporal logic), which can have an implicit or explicit representation of time. Moreover there are researchers that consider an interval-based temporal language. There are others that consider valid time (the time a fact was true in reality) and transaction time (the time the fact was stored in the database), concepts already well-known in the temporal databases field.

- Temporal direction of the formula: when the constraint language used is implicit-time temporal logic, temporal quantifiers of the formula can be related either to the past database development (past tense temporal logic) or to the future database development (future tense temporal logic). In the first case the constraint is a past-directed formula, i.e. it is expressed with past operators only (previous, sometime in the past, always in the past). In the second case it is a future-directed formula, expressed with future operators (next, sometime in the future, always in the future). If the constraint language is a type of temporal logic with explicit time, we say that the formula is bidirected. Depending on the instant of constraint evaluation and the relationship between the temporal 
variables of the formula, it can be considered a past-directed or a futuredirected formula. This feature is not applicable to methods that deal with transition constraints which use a first-order logic formula (only extended by the operators old or new). The interpretation of past/future in this case has no sense.

- Storage of historical information: this feature indicates if a method requires (or does not require) that the entire database history be stored to enforce the constraints. Constraints are usually enforced by verifying whether they are true after every database change. Dynamic constraints require checking not only the current database state, but also the entire database history. Methods that need to store all historical information in order to check the constraints are called history-based. Meanwhile some methods are carried out almost without storage of historical information. They are called history-less methods. In this case, only the minimum necessary information about the database history is stored as auxiliary relations or as historical schemas, in every database state. Thus, enforcing constraints can be done using only the current database state extended with this auxiliary information. This feature is not applicable to methods that deal with transition constraints because only two consecutive states are considered.

In what follows, we analyze several approaches with respect to the above criteria. We present the approaches grouped by the kind of the database considered, in this sequence: relational, deductive, object-oriented, deductive object-oriented, active and temporal databases. Table 1 summarizes our result.

\subsection{Approaches in the context of relational databases}

\section{The approach of Chomicki [Cho92/Cho95]}

Chomicki (1992) presents a method for dynamic constraint checking that assumes a formulation of constraints in past temporal logic with no explicit presence of time. For example, the constraint 'if a student drops out and is not subsequently reinstated, she should not be readmitted' can be written in this language as:

$\neg(\exists x)(\operatorname{admitted}(x) \wedge(\neg$ reinstated $(x)$ since dropout $(x)))$.

Chomicki's method was developed in the relational databases field. Chomicki (1992) defines his method as history-less because, although the constraints can refer to past states of the database, their checking does not require that the entire database history be stored. Instead, every database state is extended with auxiliary relations that contain the historical information necessary for checking constraints. Auxiliary relations can be implemented as materialized relational views. These relations are automatically derived from the constraint definition. In this manner, 
checking constraints after the occurrence of updates can be done using only the current extended database state.

In this approach, dynamic constraint checking resembles static constraint checking. The only differences are the construction of the extended state and the need to check constraints in the extended state. Chomicki's techniques are beneficial because dispense with the need to store the database history.

The work of Chomicki (1995) extends his previous paper (Chomicki, 1992) and presents an approach to checking real-time constraints (conditions referring to the flow of physical time, e.g., 'a renewed book should be returned within a week').

\section{The approach of Bidoit and De Amo [BDA94/BDA95]}

Very little work has been done in determining particular classes of dynamic constraints. An exception is the work of Bidoit and De Amo (1994, 1995). They introduce a class of dynamic constraints in the framework of the relational model: the dynamic algebraic dependencies (DAD) that are anteriority dependencies and subsume dynamic inclusion dependencies. These DADs allow one to express that 'if some property is true now, then in the past some other property should have hold'. They express constraints in past temporal logic with implicit time. For example, for the University database schema with the relations Stud(Name, Address) and Assist(Name,Course), they specify the constraint 'all teaching assistant should have been a student previously' by the formula:

$\forall x\left(\exists y \operatorname{Assist}(x, y) \Rightarrow \bullet^{<} \exists z \operatorname{Stud}(x, z)\right)$

In the formula, the symbol $\iota^{<}$indicates 'sometime in the past, before now'.

The main problem studied is the relationship between declarative and operational specifications of dynamic databases by means of investigating the relationship between DADs specifications and transaction schemas. They investigate in a constructive manner the definition of transaction-based specifications equivalent to DAD-constraint-based specifications.

Bidoit and De Amo (1994, 1995) enforce dynamic constraints by providing an equivalent operational specification. This approach is history-less. Their method enriches the database schema with additional relation schemas called historical schemas. Historical schemas aim at storing information about the database changes needed in order to 'check' the constraints. These historical schemas are similar to auxiliary relations introduced by Chomicki (1992).

\subsection{Approaches in the context of deductive databases}

\section{The approach of Olive [Oli91]}

Olivé (1991) proposes a method for integrity checking in deductive databases, called Internal Events Method. The method augments a database with a set of rules, called transition and internal events rules, which explicitly define the 
insertions and deletions induced by a database update. These rules depend only on the deductive rules and integrity constraints of the database. Standard SLDNF resolution can then be used to check satisfaction of integrity constraints.

Olivé (1991) deals uniformly with static and transition constraints. His method does not handle general dynamic constraints: it only deals with transitions between two successive states. Static and transition constraints are expressed by means of a closed first-order logic formula in denial form. The transition constraint formula does not contain any explicit temporal operator, e.g. the transition constraint 'the right of residence of an employee can not be deleted' is expressed as:

$\mathfrak{T i c l}(x) \leftarrow \operatorname{Emp}(x) \wedge \delta \operatorname{Rr}(x)$.

where $\operatorname{Emp}(x)$ is a fact that indicates that $x$ is a employee, $\operatorname{Rr}(x)$ indicates that $x$ has right of residence. The symbols $\imath$ and $\delta$ represent an insertion internal event and a deletion internal event that occurs in a transition of states, respectively.

The method provides a simple and uniform approach in which static and transition constraints can be defined and enforced. It has the power of methods developed so far and its implementation in Prolog does not require any metainterpreter.

\section{The approach of Plexousakis [Ple93/Ple95]}

Plexousakis (1993) proposes a method for integrity checking in deductive temporal databases. The author presents a constraint simplification method for large and semantically rich Telos knowledge bases that treats uniformly temporal and non temporal (static and dynamic) constraints. Telos (Mylopoulos et al., 1990) is a hybrid language for knowledge representation. Telos emphasizes the use of time for representing historical information about an application domain by providing two temporal dimensions for historical and belief time (also known as valid and transaction time, respectively). Constraints in Telos are specified declaratively via an explicit-time temporal logic language with interval-based time model. The constraint "an employee's salary can never decrease" is expressed as follows:

$\forall p /$ Employee $\forall s, s^{\prime} /$ Integer $\forall t 1, t 2, t 3 /$ TimeInterval $\left(\operatorname{salary}(p, s, t l, t 2) \wedge \operatorname{salary}\left(p, s^{\prime}, t 3, t 2\right) \wedge\right.$ before $\left.(t l, t 3) \Rightarrow\left(s \leq s^{\prime}\right)\right)$.

Plexousakis (1993) proposes a method that separates the constraint enforcement in two phases: a compilation phase, performed at schema definition time and an evaluation phase, performed at update time. The compilation phase performs a number of syntactic, semantic and temporal transformations to integrity constraints and deductive rules, and organizes simplified forms in a dependence graph that allows for efficient computation of implicit updates. The compile-time simplification aims at reducing the complexity of formula evaluation at run-time. 
The work of Plexousakis (1995) focuses on enhancements of the method proposed by Plexousakis (1993) with respect to the treatment of temporal constraints for arbitrary transactions. It extends the method to treat dynamic constraints in a special manner and to take into account multiple updates. Plexousakis' techniques are beneficial because focus on simplifying formulae at compile-time when performance is less critical. His method is history-based because it considers the entire history of the database.

\subsection{Approaches in the context of object-oriented (OO) databases}

\section{The approach of Lipeck et al. [LEG85/LiS87]}

Lipeck et al. (1985) discuss principles for monitoring dynamic constraints during run-time of a database. The term integrity monitoring indicates that the enforcement of constraints is undertaken by a centralized application-independent component. The authors present an ad-hoc monitoring scheme for simple temporal formulae: checking dynamic constraints is reduced to checking static constraints by exploiting the present database state and minimal knowledge about its history. They adopt a functional data model, where a schema consists of sorts of objects, functions taking arguments and delivering results of specified sorts and constraints expressed in implicit-time temporal logic. This approach incorporates temporal quantification by always, sometime, next and before. The constraint 'a car $c$ must be registered in the year of its production or in the following year' is expressed by the following formula in this language:

always $(\operatorname{produced}(c) \wedge$ year $=y \Rightarrow$ sometime registered $(c)$ before year $\geq y+2)$.

Lipeck and Saake (1987) extend the idea presented by Lipeck et al. (1985) to a large class of temporal formulae. Arbitrary non temporal parts in predicate logic are allowed as well as arbitrary nesting and logical combination of temporal quantifiers. The essential means for the monitoring proposed in this work are finite transition graphs which can be constructed from temporal formulae by utilizing an appropriate normal form. To ensure admissibility of a state sequence, the integrity monitor has to follow a corresponding path through the graph and to check certain non temporal conditions in each state. These conditions are provided as edge labels. Thus monitoring dynamic integrity is reduced to a controlled checking of static constraints.

The work of Lipeck and Saake (1987) is close to Chomicki's work (Chomicki, 1992; 1995) because it also deals with the problem of history-less checking of dynamic constraints in the presence of arbitrary updates. However, Lipeck and Saake (1987) work in the future fragment of temporal logic and Chomicki (1992, 1995), in the past temporal logic. 


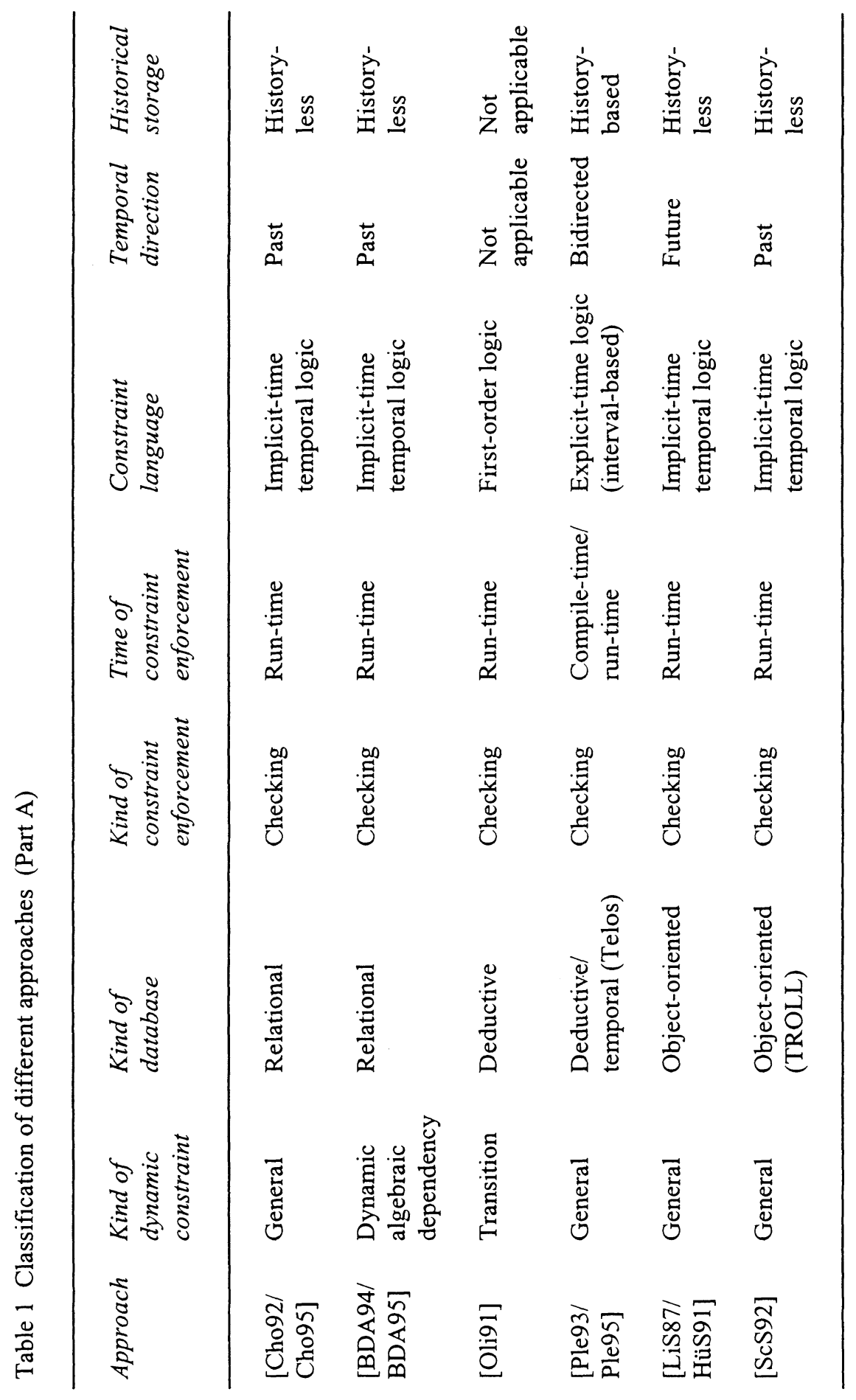




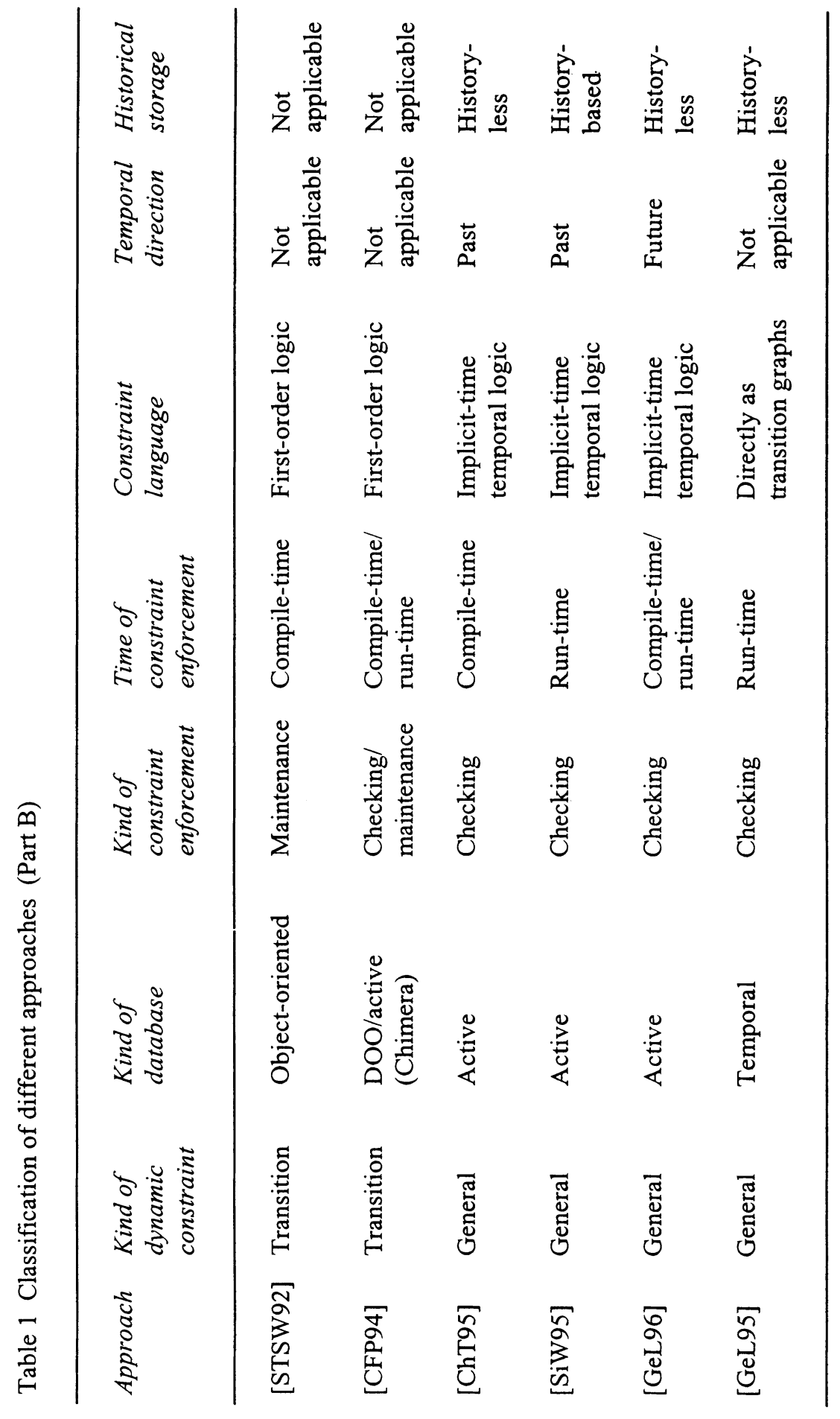


The approach of Saake et al. [HüS91/ScS92]

Researches of Saake et al. (Hülsmann and Saake, 1991; Schwiderski and Saake, 1992) continue Lipeck's work (Lipeck and Saake, 1987) in dynamic integrity monitoring.

Hülsmann and Saake (1991) extend the already known techniques for minimizing the stored information for a single substitution of the free constraint variables using transition graph construction. They present a formalism to restrict the storing of historical information to those object/data combinations where this is necessary to evaluate the constraint. Moreover, by handling sets of such combinations instead of single combinations it further minimizes the storage demands. This work adopts the same characterizing features of the work of Lipeck and Saake (1987). See in Table 1 that both approaches are grouped together in a same line.

Schwiderski and Saake (1992) pursue the approach taken by Hülsmann and Saake (1991) to presenting another method for monitoring temporal permissions during database run-time. The difference is the monitoring of past-directed dynamic constraints in the approach of Schwiderski and Saake (1992). In this paper, temporal permissions are used as enabling conditions for the occurrence of specific state-changing operations in the TROLL language (Jungclaus et al., 1996). TROLL aims at integrating a declarative, logic-based style of system specification with structuring mechanisms known from semantic and object-oriented data models. In TROLL, descriptions of the static and dynamic aspects of entities are integrated into object descriptions, having a formal semantics in terms of a translation into a implicit-time temporal logic.

Because Schwiderski and Saake (1992) deal with past-directed constraint evaluation, they use the transition graphs in a reverse direction. The initial idea of these graphs (Lipeck and Saake, 1987) was to represent future-directed constraints. The method of Schwiderski and Saake (1992) also avoids storing the whole past life cycle of the database. This approach resembles the work of Chomicki (1992) because both approaches are history-less and deal with past temporal logic. However, Schwiderski and Saake (1992) use an explicit dependency of temporal conditions and update operations so that the monitoring can be optimized in several ways.

\section{The approach of Schewe et al. [STSW92]}

Schewe et al. (1992) address the integrity enforcement problem with respect to static and transition constraints. Their analysis is based on object-oriented databases with a clear distinction between values and objects. They use formulae in first-order logic to express both static and transition constraints.

This paper adopts the approach of modifying a user's transaction by replacing the methods that update objects with rewritten ones, which satisfy the constraints. They give formally defined general proof obligations for static and transition 
consistency and discuss that using a general purpose theorem prover to prove these formulae gives a verification based approach to consistency.

However, as the verification approach does not help in writing consistent methods, their idea is to replace each update operation (methods in the OODM) by a Greatest Consistent Specialization (GCS). A GCS is defined as a derived operation that augments the original one to make it compliant to a single constraint. The GCS of an update operation represents a conditional update that guarantees consistency. The drawback of the approach is that GCSs must be built manually.

In this method, integrity enforcement can be performed completely at compiletime. It supports integrity maintenance because the GCS can add to the original operation extra updates, similar to repairing actions (Fraternali and Paraboschi, 1993). Because the method deals only with transition (not general dynamic) constraints, the criterion temporal direction (in Table 1) is not applicable in this case. We can not consider past/future interpretation for this kind of formulae. Moreover, the criterion historical storage is not applicable as well because only two consecutive states are considered.

\subsection{Approaches in the context of deductive object-oriented (DOO) databases}

The approach of Ceri et al. [CFP94]

Ceri et al. (1994a) present a proposal for constraint enforcement in Chimera (Ceri and Manthey, 1994). Chimera introduces an attempt towards integrating active, deductive and object-oriented data modeling and manipulation techniques within a unified framework. Static and transition constraints in Chimera are expressed by first-order logic formulae as deductive rules in a denial form. Transition constraints are introduced for the purpose of monitoring state changes and they are supported by means of references to past database states within the body of rules defining integrity constraints. A special predicate old can be applied to attribute terms in order to denote the past state of the objects. Syntactically, transition constraints are recognized for this special predicate. For example, the following constraint states that 'the salary of an employee should not grow more than 50\%':

add constraint increaseExcess for employee

as increaseExcess $($ Self $)<-$ Self.salary $>$ old (Self.salary) $* 1.5$.

Ceri et al. (1994a) advocate a flexible approach for Chimera, where constraints can be tackled at different levels of sophistication:

- At the simplest level, constraints are expressed declaratively through deductive rules, whose head defines a constraint predicate that can be used by the 
transaction supplier to query constraint violations. Integrity is then enforced manually by the transaction supplier.

- At the next level, constraints are encoded as triggers, activated by any event likely to violate the constraint. Their precondition tests for the actual occurrence of violations. If a violation is detected, then the rule issues a rollback command. These rules (called abort rules) can be syntactically generated from declarative constraints. At this level, only integrity checking is considered because repair actions are not undertaken in case of violations.

- At the most sophisticated level, constraints are encoded as triggers having the same precondition and triggering events as abort rules. Their action part contains database manipulations for repairing constraint violations. These rules are called maintenance rules. At this level, integrity maintenance is performed by permitting an integrity repairing strategy where, instead of aborting transactions, changes are applied to the database in order to reach a consistent situation. This approach adapts the work proposed by Ceri et al. (1994b) for relational databases.

\subsection{Approaches in the context of active databases}

\section{The approach of Chomicki and Toman [ChT95]}

The work of Chomicki and Toman (1995) pursues the approach taken by Chomicki (1992) and proposes a general architecture for implementing dynamic constraints by compiling them into a set of active DBMS rules. These rules guarantee enforcement of the constraints on the underlying database.

The advantages of this architecture are demonstrated on a particular temporal constraint compiler. This compiler allows automatic translation of constraints formulated in past temporal logic into rules of an active DBMS. During the translation process all the formulas are checked for the safe evaluation property. The result is a set of SQL statements that includes all the necessary rules needed for enforcing the original constraints. Also several optimization steps are performed for the proposed architecture.

In several aspects, this method resembles Chomicki (1992). It is also a historyless method; it performs dynamic constraint checking and it also specifies constraints by an implicit-time temporal logic. Different from Chomicki (1992) which proposes a run-time method, the approach of Chomicki and Toman (1995) proposes a method that performs completely at compile-time. There is no need for an additional run-time constraint monitor. In this respect, the utilization of active rules has an important advantage compared with other methods. When the rules are activated, all updates to the database that violate any of the constraints are automatically rejected. 


\section{The approach of Sistla and Wolfson [SiW95]}

Sistla and Wolfson (1995) use past temporal logic for specifying conditions and events in the rules for active database system. Basically, the language used consists of a regular query language augmented with a set of temporal operators, such as Since, Previously, Lasttime and Throughout_the_Past. However, instead of standard first-order quantifiers, they use nonstandard freeze quantifiers (a freeze quantifier binds a variable to a single value or a relation instance in a given temporal context). They present an algorithm for incremental evaluation of temporal conditions and a new framework for processing temporal (static and dynamic) constraints.

This evaluation is incremental in the sense that when a new database state is created as a result of an update, the algorithm only considers the changes in the new database state in order to determine if the condition is satisfied, instead of considering the whole database history. This method can use either valid time or transaction time. It also is capable of expressing real-time conditions as Chomicki (1995) but using a distinguished variable time.

According to Chomicki (1995), Sistla and Wolfson's method does not provide bounded history encoding (history-less) and the amount of historical information kept grows with the age of the database. This may be a serious problem because the method postulates discarding all this information after a trigger fires.

\section{The approach of Gertz and Lipeck [GeL96]}

The approach of Gertz and Lipeck (1996) is based on Lipeck's previous works on monitoring schemes for dynamic integrity constraints (Lipeck et al., 1985; Lipeck and Saake, 1987) and on rules for transforming constraints into transaction specification (Lipeck, 1990). Gertz and Lipeck (1996) consider an active database and present how to generate triggers for monitoring integrity from dynamic constraint formulae. These formulae are specified in temporal logic with operators like always, sometime, next, before and until, which can equivalently be transformed into transition graphs. They also present how to refine rules according to graph properties to get more efficient triggers.

In this method only the minimum information needed for monitoring is kept in the object situations corresponding to nodes of transition graphs. Similar to Chomicki's approach (Chomicki, 1992; Chomicki and Toman, 1995) every database state is augmented with auxiliary relations which are used to check a dynamic constraint. Different from Chomicki's approach, Gertz and Lipeck (1996) use only one auxiliary relation per constraint to represent the current situation of each object. Moreover, they consider optimizations and simplification of triggers. The further simplifications to improve efficiency on integrity checking rely on identifying objects not affected by the transaction and not involved in potential constraint violations since corresponding condition instances are invariant.

Gertz and Lipeck (1996) also discuss the integration of this approach into the database design. In this way, the specification of static and dynamic constraints 
and their transformation into integrity enforcement triggers becomes a main design task. Moreover, they discuss that the triggers (whose action parts consist only of a simple rollback of the triggering transaction) should at least be enriched (at design time) by actions and procedures which visualize the detected violations of a constraint and violating tuples.

\subsection{Approaches in the context of temporal databases}

\section{The approach of Gertz and Lipeck [GeL95]}

Gertz and Lipeck (1995) incorporate the monitoring of integrity constraints proposed by Lipeck et al. (1985) and Lipeck and Saake (1987) into temporal databases. In this approach, Gertz and Lipeck (1995) do not want to emphasize temporal logic as a constraint specification language. They use directly transition graphs as descriptions of dynamic constraints because their objective is to study a class of integrity constraints dealing with object life cycles over the course of time. Such transition graphs describes all the admissible life cycles of objects with respect to the underlying constraint. The graph nodes represent the minimal historical information needed for monitoring life cycles with respect to this constraint.

The paper presents an algorithmic scheme for monitoring such constraints in temporal databases which efficiently support the concept of valid time. They assume a temporal data model which supports storing information about the past, present and future. The keys to efficient monitoring lie in storing life cycle situations with respect to constraints, in utilizing the property of graphs being iteration-invariant, and in using basic operations of temporal databases to determine validity intervals.

In this method, integrity checking is performed at run-time. Because the method specifies constraints directly as transition graphs, the criterion temporal direction in Table 1 (past or future) is not applicable in this case.

\section{CONCLUSION}

We have presented a review of relevant approaches to dynamic integrity constraint enforcement. Many papers in the literature have presented a survey of this topic for static constraints. However, to the best of our knowledge, there are not a published survey about dynamic constraint enforcement.

First we have introduced some general concepts in database integrity enforcement and presented some methods to enforcing static constraints. Then we have discussed several methods developed for dynamic constraint enforcement. Finally we have classified and compared the illustrated methods with respect to some characterizing features. 
We have concluded that most methods use a temporal logic language with implicit time for specifying dynamic constraints. Such approaches incorporate temporal quantification by operators as always, sometime, before, next, previously and since. In some approaches these constraints are past-directed formulae while in others, constraints are specified by future-directed formulae. We have also concluded that the majority of authors agree that it is important to avoid storing the whole history of the database. In this case, methods developed are called historyless and only the minimum historical information needed for the constraint enforcement is stored. Also, most approaches propose checking instead of maintenance of the database integrity, i.e. they abort or rollback the transaction in case of violations and repair actions are not undertaken. Moreover, the majority of methods perform constraint enforcement completely at run-time.

\section{Acknowledgements}

The author is very grateful to Maria Ribera Sancho, Antoni Olivé, Joan Antoni Pastor, Enric Mayol and the anonymous reviewers for their useful comments and suggestions.

This work has been partially supported by CICYT (Spain), project TIC95-0735. The author is supported by a scholarship from CAPES (MEC/Brazil).

\section{REFERENCES}

Asirelli, P., Inverardi, P. and Plagenza, G. (1996) Integrity constraints as views in deductive databases. Proc. of the 6th Int. Workshop on Foundations of Models and Languages for Data and Objects: Integrity in Databases, Schloss Dagstuhl, Germany.

Bertino, E., Catania, B. and Bressan, S. (1997) Integrity constraint checking in Chimera. Proc. of the 2nd Int. Workshop on Constraints Database Systems, Delphi, Greece, 160-86.

Bidoit, N. and De Amo, S. (1994) Contraintes dynamiques d'inclusion et schémas transactionnels. Ingénierie des systèmes d'information, 2(1), 83-113.

Bidoit, N. and De Amo, S. (1995) A first step towards implementing dynamic algebraic dependencies. Proc. of the 5th Int. Conference on Database Theory, Prague, Czech Republic, 308-21.

Bry, F., Decker, H. and Manthey, R. (1988) A uniform approach to constraint satisfaction and constraint satisfiability in deductive databases. Proc. First Int. Conference on Extending Database Technology, Venice, Italy, 488-505.

Bry, F., Manthey, R. and Martens, B. (1990) Integrity verification in knowledge bases. ECRC report D.2.1.a, Munich, Germany.

Caseau, Y. (1991) Constraints in an object-oriented deductive database. Proc. of the 2nd Int. Conference on Deductive and Object-Oriented Databases, Munich, Germany, 292-311. 
Ceri, S., Fraternali, P. and Paraboschi, S. (1994a) Constraint management in Chimera. Data Engineering Bulletin, 17(2), 4-8.

Ceri, S., Fraternali, P., Paraboschi, S. and Tanca, L. (1994b) Automatic generation of production rules for integrity maintenance. ACM Transactions on Database Systems, 19(3), 367-422.

Ceri, S. and Manthey, R. (1994) Chimera: A model and language for active DOOD systems. Proc. of the 2nd International East/West Database Workshop, Klagenfurt, Austria, 3-16.

Ceri, S. and Widom, J. (1990) Deriving production rules for constraint maintenance. Proc. of the I6th Int. Conference on Very Large Data Bases, Brisbane, Australia, 566-77.

Chomicki, J. (1992) History-less checking of dynamic integrity constraints. Proc. 8th IEEE Int. Conference on Data Engineering, Phoenix, Arizona, 557-64.

Chomicki, J. (1995) Efficient checking of temporal integrity constraints using bounded history encoding. ACM Transactions on Database Systems, 20(2), 149-86.

Chomicki, J. and Toman, D. (1995) Implementing temporal integrity constraints using an active DBMS. IEEE Transactions on Knowledge and Data Engineering, issue on Temporal and Real-Time Databases, 7(4), 566-82.

Decker, H. (1986) Integrity enforcement on deductive databases. Proc. First Int. Conference on Expert Database Systems, Charleston, South Carolina, 271-85.

Florentin, J. J. (1974) Consistency auditing of databases. The Computer Journal, $17(1), 52-8$.

Formica, A., Missikoff, M. and Terenzi, R. (1994) Constraint satisfiability in object-oriented databases. Proc. of the 2nd Int. East/West Database Workshop, Klagenfurt, Austria, 48-60.

Fraternali, P. and Paraboschi, S. (1993) A review of repairing techniques for integrity maintenance. Proc. of the First Int. Workshop on Rules in Database Systems, Edinburgh, Scotland, 333-46.

Gertz, M. and Lipeck, U. W. (1995) 'Temporal' integrity constraints in temporal databases. Proc. of the Int. Workshop on Temporal Databases, Zurich, Switzerland, 72-92.

Gertz, M. and Lipeck, U. W. (1996) Deriving optimized integrity monitoring triggers from dynamic integrity constraints. Data \& Knowledge Engineering, 20(2), 163-93.

Grefen, P. and Apers, P. (1993) Integrity control in relational database systems - an overview. Data \& Knowledge Engineering, 10(2), 187-223.

Gustafsson, M. R., Karlsson, T. and Bubenko, J. A. (1982) A declarative approach to conceptual information modeling, in Information systems design methodologies: A comparative review (eds. T. W. Olle, H. G. Sol, and A. A. Verrijn-Stuart), North-Holland, 93-142.

Henschen, L. J., McCune, W. W. and Naqvi, S. A. (1984) Compiling constraintchecking programs from first-order formulas, in Advances in Database 
Theory, Vol. 2 (eds. H. Gallaire, J. Minker and J.-M. Nicolas), Plenum Press, New York, 145-69.

Hsu, A. and Imielinski, T. (1985) Integrity checking for multiple updates. Proc. of the ACM-SIGMOD Int. Conf. Management of Data, Austin, Texas, 152-68.

Hülsmann, K. and Saake, G. (1991) Theoretical foundations of handling large substitution sets in temporal integrity monitoring. Acta Informatica, 28(4), 365-407.

Jeusfeld, M. and Jarke, M. (1991) From relational to object-oriented integrity simplification. Proc. of the 2nd Int. Conference on Deductive and ObjectOriented Databases, Munich, Germany, 460-77.

Jungclaus, R., Saake, G., Hartmann, T. and Sernadas, C. (1996) TROLL - A language for object-oriented specification of information systems. $A C M$ Transactions on Information Systems, 14(2), 175-211.

Karadimce, A. P. and Urban, S. D. (1993) A framework for declarative updates and constraint maintenance in object-oriented databases. Proc. of the 9th IEEE Int. Conference on Data Engineering, Vienna, Austria, 391-8.

Kowalski, R., Sadri, F. and Soper, P. (1987) Integrity checking in deductive databases. Proc. 13th Int. Conference on Very Large Data Bases, Brighton, England, 61-9.

Lee, S. J. and Ling, T. W. (1996) Further improvement on integrity constraint checking for stratifiable deductive databases. Proc. of the 22th Int. Conference on Very Large Data Bases, Bombay, India, 495-505.

Lipeck, U. W. (1990) Transformation of dynamic integrity constraints into transaction specifications. Theoretical Computer Science, 76, 115-42.

Lipeck, U. W., Ehrich, H.-D. and Gogolla, M. (1985) Specifying admissibility of dynamic database behaviour using temporal logic. Proc. of the IFIP Working Conference on Theoretical and Formal Aspects of Information Systems, Amsterdam, Holland, 145-57.

Lipeck, U. W. and Saake, G. (1987) Monitoring dynamic integrity constraints based on temporal logic. Information Systems, 12(3), 255-69.

Lloyd, J. W., Sonenberg, E. A. and Topor, R. W. (1987) Integrity constraint checking in stratified databases. Journal of Logic Programming, 4(4), 331-43.

Moerkotte, G. and Lockemann, P. C. (1991) Reactive consistency control in deductive databases. ACM Transactions on Database Systems, 16(4), 670-702.

Motro, A. (1989) Integrity $=$ validity + completeness. ACM Transactions on Database Systems, 14(4), 480-502.

Mylopoulos, J., Borgida, A., Jarke, M. and Koubarakis, M. (1990) Telos: Representing knowledge about information systems. ACM Transactions on Information Systems, 8(4), 325-62.

Nicolas, J.-M. (1982) Logic for improving integrity checking in relational data bases. Acta Informatica, 18(3), 227-53. 
Nicolas, J.-M. and Yazdanian, K. (1978) Integrity checking in deductive data bases, in Logic and Data Bases (eds. H. Gallaire, and J. Minker), Plenum Press, New York, 325-44.

Olivé, A. (1991) Integrity constraints checking in deductive databases. Proc. of the 17th Conference on Very Large Data Bases, Barcelona, Spain, 513-23.

Olivé, A. (1995) Integrity constraints specification. Technical report LSI, Universitat Politècnica de Catalunya, Barcelona, Spain.

Pacheco, M. A. and Sancho, M. R. (1997) Especificación de restricciones de integridad en el sistema ROSES. Technical report LSI-97-19-R, Universitat Politècnica de Catalunya, Barcelona, Spain.

Palopoli, L. and Torlone, R. (1992) Maintaining integrity constraints on complex objects. Proc. of the 3rd European Research Consortium for Informatics and Mathematics on Updates and Constraints Handling in Advanced Databases Systems, Pisa, Italy, 10-24.

Pastor, J. A. and Olivé, A. (1994) An approach to the synthesis of update transactions in deductive databases. Proc. of the 5th Int. Conference on Information Systems and Management of Data, Madras, India, 93-110.

Plexousakis, D. (1991) Semantic integrity enforcement in knowledge bases. $\mathrm{PhD}$. qualifying examination paper, Department of Computer Science, University of Toronto, Canada.

Plexousakis, D. (1993) Integrity constraint and rule maintenance in temporal deductive knowledge bases. Proc. of the 19th Int. Conference on Very Large Data Bases, Dublin, Ireland, 146-57.

Plexousakis, D. (1995) Compilation and simplification of temporal integrity constraints. Proc. of the 2nd Int. Workshop on Rules in Database Systems, Athens, Greece, 260-76.

Qian, X. and Waldinger, R. (1988) A transaction logic for database specification. Proc. of the ACM-SIGMOD Int. Conference on Management of Data, Chicago, USA, 243-50.

Sancho, M. R. and Olivé, A. (1993) The ODISSEA approach to the design of information systems from deductive conceptual models. Technical report LSI93-16-R, Universitat Politècnica de Catalunya, Barcelona, Spain.

Schewe, K.-D., Thalheim, B., Schmidt, J. W. and Wetzel, I. (1992) Integrity enforcement in object-oriented databases. Proc. of the 4th Int. Workshop on Foundations of Models and Languages for Data and Objects, Volkse, Germany, 181-206.

Schwiderski, S. and Saake, G. (1992) Monitoring temporal permissions using partially evaluated transition graphs. Proc. of the 4th Int. Workshop on Foundations of Models and Languages for Data and Objects, Volkse, Germany, 196-217.

Siebes, A. and Van der Voort, L. (1992) Active objects and constraint enforcement. Extended abstract. Proc. of the 3rd European Research 
Consortium for Informatics and Mathematics on Updates and Constraints Handling in Advanced Databases Systems, Pisa, Italy, 1-9.

Sistla, A. P. and Wolfson, O. (1995) Temporal conditions and integrity constraints in active database systems. Proc. of the ACM-SIGMOD Int. Conference on Management of Data, San Jose, California, 269-80.

Stonebraker, M. (1975) Implementation of integrity constraints and views by query modification. Proc of the ACM-SIGMOD Int. Conference on Management of Data, 65-78.

Teniente, E. and Olivé, A. (1995) Updating knowledge bases while maintaining their consistency. The VLDB Journal, 4(2), 193-241.

Urban, S. D. and Delcambre, L. M. L. (1990) Integrity analysis: a design process for specifying operations on objects. IEEE Transactions on Knowledge and Data Engineering, 2(4), 391-400.

Wallace, M. (1991) Compiling integrity checking into update procedures. Proc. of the 12th Int. Conference on Artificial Intelligence, Sydney, Australia, Vol. 2, 903-8.

Widom, J., Gupta, A., Sagiv, Y. and Ullman, J. D. (1994) Validating constraints with partial information: Research overview. Proc. of the Sth Int. Workshop on the Deductive Approach to Information Systems and Data Bases, Aiguablava, Spain, 375-85.

Wüthrich, B. (1993) On updates and inconsistency repairing in knowledge bases. Proc. of the 9th IEEE Int. Conference on Data Engineering, Vienna, Austria, 608-15.

\section{BIOGRAPHY}

Maria Amélia Pacheco e Silva received the BS degree in engineering and the MS degree in computer science from the Federal University of Uberlandia (Brazil) in 1983 and 1992, respectively. She worked as computer programmer and as systems analyst at Federal University of Uberlandia from 1979-82 and 1983-93, respectively. She is currently working towards her Ph.D. degree in computer science at the Technical University of Catalonia (Spain). Her areas of interest include conceptual models, methodologies and tools for information system analysis, database systems and integrity constraint management. 